\title{
Intoxicación aguda por plaguicidas
}

Jaime Jesús Durán-N ah, M.C ., M. en C., ${ }^{(1)}$ Julián C ollí-Q uintal, M.C. ${ }^{(2)}$

\section{Durán-Nah JJ, Collí-Quintal J. Intoxicación aguda por plaguicidas. Salud Publica Mex 2000;42:53-55.}

\begin{abstract}
Resumen
Objetivo. D escribir la epidemiología de la intoxicación aguda por plaguicidas (IAP) en el Hospital G eneral O 'H orán de Mérida, Yucatán, México. Material y métodos. Se revisaron los expedientes de 33 pacientes $\geq 13$ años, tratados en una unidad de cuidados intensivos (U C I), entre 1994 y 1998. Se recopilaron variables demográficas y clínicas relevantes, y se aplicó estadística descriptiva. Resultados En la población estudiada predominó el sexo masculino (82\%) del medio rural $(70 \%)$, y la edad media fue de $34 \pm 15.8$ años. El intento de suicidio fue causa frecuente de IAP $(79 \%)$, y en $33 \%$ de los casos la intoxicación se produjo por la utilización de organo fosforados. La mortalidad fue de $12 \%$. Conclusiones. La IAP fue baja entre los sujetos del medio rural, por lo que los resultados de este estudio parecen no reflejar la realidad del problema. Es importante añadir que los plaguicidas son utilizados con relativa frecuencia para el intento de suicidio.
\end{abstract}

Palabras clave: plaguicidas/toxicidad; intento de suicidio; México
Durán-Nah JJ, Collí-Quintal J. Acute poisoning from pesticides. Salud Publica Mex 2000;42:53-55.

\begin{abstract}
A bstract
Objective To describe the epidemiologic pattern of acute pesticide poisoning (APP) in a general hospital in Merida, Yucatan, Mexico. Material and methods. From 1994 to 1998, 33 patients 13 years of age or older with diagnosis of APP were studied. Descriptive statistics were used to analyze information. Results. Males were frequently affected $(82 \%)$, specially those coming from rural areas $(60 \%)$. The mean age of the group was $34 \pm 15.8$ years. In $79 \%$ of the cases, pesticides were used to commit suicide and $33 \%$ of poisoning cases were due to organo phospate pesticides. The mortality rate was $12 \%$. Conclusions. In this small sample, acute poisoning from pesticides in the agricultural setting may be underestimated, since it was less frequent than in the general population. APP was more commonly used by indigent people to commit suicide.
\end{abstract}

Key words: pesticide/toxicity; suicide attempt; Mexico

toriales dedicadas a la agricultura y la horticultura, donde se utilizan variados tipos de plaguicidas. ${ }^{4} \mathrm{Se}$ esperaría que los casos de intoxicación aguda se produjeran frecuentemente en gente que trabaja con ellos.

La Unidad de Cuidados Intensivos (UCI) del Hospital General O'Horán (HGOH), en la ciudad de Mérida, Yucatán, recibe con relativa frecuencia casos de IAP. Describiremos las características demográficas y clínicas de una serie de casos atendidos durante cinco años en dicho servicio.

(1) Servicio de Medicina Interna, Hospital General O 'H orán, Mérida, Yucatán, México.

(2) Médico en práctica privada, Mérida,Yucatán, México.

Fecha de recibido: 29 de octubre de 1999 - Fecha de aprobado: 11 de noviembre de 1999 Solicitud de sobretiros: Jaime Jesús Durán-N ah, D epartamento de Medicina Interna, Hospital General 0 'Horán. A venida Itzaes X Jacinto Canek, 97000 Mérida,Yucatán, México. 


\section{Material y métodos}

Se revisaron 33 expedientes de pacientes $\geq 13$ años de edad con diagnóstico de IAP, egresados de la UCI del HGOH, entre 1994 y 1998. Se recopilaron variables demográficas y clínicas relevantes, y se aplicó estadística descriptiva. A algunas medias obtenidas se les aplicó la prueba $t$ de Student para una muestra o para muestras independientes, con intervalos de confianza (IC) de 99 y 95\%, respectivamente.

\section{Resultados}

La edad grupal de los casos de IAP varió de 16 a 70 años, $\bar{X}=34 \pm 15.8$ (IC99\% 26.4-41.6). Veintisiete sujetos $(82 \%)$ eran hombres, y seis $(18 \%)$, mujeres, procedentes en su mayor parte del medio rural $(70 \%)$. Veinte pacientes $(61 \%)$ desempeñaban ocupaciones no relacionadas con el uso de plaguicidas, en tanto que 13 (39\%) eran campesinos. La causa de exposición fue el intento de suicido en 26 casos (79\%) y accidente laboral, en siete $(21 \%)$. La intoxicación por organofosforados se presentó en 11 sujetos (33\%); por carbamatos, en nueve $(27 \%)$; por compuestos bipiridílicos (paraquat), en seis $(18 \%)$, y por rodenticida, en uno (3\%). Por último, seis de los pacientes (18\%) señalaron desconocer el tipo de plaguicida que los había intoxicado. En el cuadro I se presentan los plaguicidas de uso más frecuente en el campo, al menos en la región noreste del estado de Yucatán, y que difieren, en parte, de los más utilizados por los pacientes que llegan al $\mathrm{HGOH}$.

La exposición fue por ingestión en 25 casos $(76 \%)$; por contacto dérmico, en cinco $(15 \%)$, y por inhalación, en dos $(6 \%)$. Un paciente se administró el plaguicida por vía intramuscular en el brazo.

El cuadro clínico fue pleomórfico; la miosis, la broncorrea y la insuficiencia respiratoria fueron frecuentes. Otros datos relevantes se muestran en el cuadro II. Se consideraron con intoxicación grave $15(45 \%)$ casos; con moderada, ocho (24\%), y con leve, $10(30 \%)$. Diez y seis pacientes (48\%) tuvieron complicaciones, entre las que predominaron las respiratorias (neumonía) y las metabólicas (acidosis). Ocho sujetos (24\%) llegaron al hospital en estado de coma, siete de los cuales presentaron paro respiratorio durante su valoración clínica inicial. El tiempo de estancia en la UCI varió entre 1 y 17 días, con una mediana de uno y un rango intercuartílico de 14 . Para el grupo con complicaciones, la estancia fue de 1 a 17 días, $\bar{X}=7 \pm 6.5$, y para el grupo sin ellas, fue de 1 a 4 días, $\bar{X}=1.5 \pm 1$. La diferencia entre ambos fue significativa (prueba $t$ de Student para muestras independientes, $p=0.05$, IC $95 \%$

\section{Cuadro I \\ Diez plaguicidas de uso más frecuente en el NORESTE de YuCatán. MÉxico, 1998}

\begin{tabular}{lllr}
$\begin{array}{l}\text { Nombre } \\
\text { químico }\end{array}$ & \multicolumn{1}{c}{$\begin{array}{c}\text { Grupo } \\
\text { químico }\end{array}$} & \multicolumn{1}{c}{$\begin{array}{l}\text { Tipo de } \\
\text { acción }\end{array}$} & $\begin{array}{r}\text { Cantidad } \\
\text { utilizada* }\end{array}$ \\
Mancozab & Tiocarbamato & Fungicida & 9640 \\
\hline Captán & Dicarboximida & Fungicida & 6750 \\
\hline Paraquat & Bipiridílico & Herbicida & 5926 \\
\hline Oxicloruro de cobre & Cúprico & Fungicida & 4556 \\
\hline Metamidofós & O F & Insecticida & 4209 \\
\hline Benomilo & Carbamato & Insecticida & 3000 \\
\hline Paraquat-diquat & Bipiridílico & Herbicida & 2900 \\
\hline Clorotalonilo & Benzonitrilo & Herbicida & 2440 \\
\hline Dimetoato & O F & Insecticida & 1433 \\
\hline Endosulfán & Clorado & Insecticida & 975 \\
* Litros o kg & & & \\
* O F:O rganofosforado & & &
\end{tabular}

Fuente: Vázquez-Fonseca J, Alvarado-M ejía J, González-N avarrete. Plaguicidas de uso común durante 1998 en la región noreste del estado de Yucatán. Mérida: D epartamento de Investigación-Facultad de MedicinaUniversidad Autónoma de Yucatán. D ocumento no publicado

1.76-8.5). Fallecieron cuatro pacientes $(12 \%)$ con edades de entre 18 a 70 años, $\bar{X}=39 \pm 23.7$, todos pertenecientes al grupo que llegó al hospital en estado de coma.

\section{Discusión}

En el estado de Yucatán la IAP es poco frecuente. ${ }^{5,6} \mathrm{En}$ la presente serie, la mayoría de los pacientes sufrió intoxicación intencional, lo que se presentó predominantemente entre varones jóvenes del medio rural, con ocupaciones poco remuneradas. Lo observado en este estudio tiene cuando menos dos connotaciones: una de ellas está relacionada con el suicidio, ${ }^{7,8}$ y la otra, con problemas sociodemográficos, ${ }^{9}$ (como baja escolaridad, y ocupación con ingreso económico bajo), ambas íntimamente relacionadas entre sí.

Los casos de IAP pueden ser de hasta un millón por año en los países en desarrollo., ${ }^{2,10}$ Algunos de los argumentos que se dan para explicar esta cifra son variados; entre ellos, se cita el uso de ropa o equipo inadecuados, la escasa preparación, el incumplimiento de las normas correspondientes y la falta de una supervisión experta en el manejo, el uso y la aplicación de los plaguicidas, así como la alta toxicidad de algunos de estos compuestos, los que incluso han sido prohibidos en países industrializados. ${ }^{10-12}$ Varios de estos argumentos pueden aplicarse a los casos de intoxicación acci- 


\section{Cuadro II \\ Cuadro clínico al ingresar de pacientes con INTOXICACIÓN AGUDA POR PLAGUICIDAS. HOSPITAL General O’Horán, Yucatán, México, 1994-1998}

\begin{tabular}{lcc} 
Dato clínico* & Frecuencia & Porcentaje \\
Miosis & 21 & 64 \\
\hline Broncorrea & 21 & 64 \\
\hline Insuficiencia respiratoria & 21 & 64 \\
\hline Irritabilidad & 19 & 58 \\
\hline Fasciculaciones & 19 & 58 \\
\hline Salivación & 18 & 55 \\
\hline Diaforesis & 18 & 55 \\
\hline Confusión & 13 & 39 \\
\hline Contracturas musculares & 13 & 39 \\
\hline Vómito & 12 & 36 \\
\hline Bradicardia & 12 & 36 \\
\hline Debilidad muscular & 11 & 33 \\
\hline Hiperreflexia & 7 & 21 \\
\hline Paro respiratorio & 7 & 21 \\
\hline Coma & 7 & 21 \\
\hline Taquicardia & 6 & 18 \\
\hline Diarrea & 6 & 18 \\
\hline Relajación de esfínteres & 5 & 15 \\
\hline Lagrimeo & 4 & 12 \\
\hline Rinorrea & 3 & 9 \\
\hline Convulsiones & 2 & \\
\hline Lesiones en vía digestiva & $\mathrm{D}$ & \\
\hline Irritación ocular & $\mathrm{D}$ & \\
\hline
\end{tabular}

* Un mismo paciente pudo presentar varios al mismo tiempo ND: no documentado

dental en Yucatán, estado en el que se diversifica cada vez más el tipo de plaguicidas utilizados., ${ }^{4, *}$

De 10000 a 20000 decesos ocurren anualmente como consecuencia de la IAP a escala mundial, cifra que puede llegar a incrementarse hasta diez veces más, especialmente en los países en desarrollo. ${ }^{2,10,11} \mathrm{La}$ frecuencia es constante en muchos países. En la presente serie, y aunque en distintas circunstancias, la mortalidad fue comparable con la citada por Carvalho para el país. ${ }^{10}$

El cuadro clínico fue variado, aunque acorde con lo referido por Vance. ${ }^{1}$ Llamó la atención la ausencia de datos clínicos que caracterizan la exposición aguda por paraquat, ${ }^{1}$ aunque probablemente ello se deba a que éstos no se buscaron intencionalmente. Durante la evolución clínica, se presentaron todo tipo de com-

\footnotetext{
* Vázquez-Fonseca J, Alvarado-Mejía J y González-Navarrete L. Plaguicidas de uso común durante 1998, en la región noreste del estado de Yucatán. Datos inéditos. Departamento de Investigación, Facultad de Medicina, Universidad Autónoma de Yucatán. Mimeografiado.
}

plicaciones que prolongaron significativamente la estancia hospitalaria en este grupo de pacientes. La mortalidad se dio en aquellos que ingresaron graves al hospital, desenlace que concuerda al menos con la intoxicación por organofosforados. ${ }^{1}$

Los casos de IAP en el HGOH no son frecuentes en gente que trabaja con ellos. No obstante, es probable que su frecuencia sea mayor a la que observamos, especialmente por aquellos casos clínicamente leves que no llegan al hospital. Llama la atención la frecuencia de intento de suicidio mediante el uso de estos compuestos.

\section{Agradecimientos}

Agradecemos a las autoridades médicas y administrativas del Hospital General O’Horán y a los jefes del Departamento Clínico, así como al personal médico y administrativo de los servicios de Medicina Interna y Archivo Clínico, ya que su valiosa cooperación permitió la realización del presente trabajo.

\section{Referencias}

1.Vance MV. Pesticides. En: Rose P, Barkin RM, Braen GR, Levy RG, D ailey RH, Marx JA et al. Emergency medicine. Concepts and clinical practice. $3 a$. edición. St Louis (MO): Mosby Year Book, 1992;vol..Il:2630-2641.

2. Klein-Schwartz W, Smith GS. Agricultural and horticultural chemical poisoning. Mortality and morbidity in the United States. Ann Emerg Med 1997:29:232-238.

3. 0 rtega-C eseña J, Carreón-ValenciaT, López-C arrillo L,C hávez-Ayala R, Hernández-Avila M. La investigación en México sobre el impacto en la salud por los contaminantes químicos ambientales. Salud Publica Mex 1993;35:585-591.

4. Alvarado-Mejía J, C obos-G ascaV, González-N avarrete L. Insecticidas y herbicidas de mayor uso en los horticultores de Yucatán, implicaciones a la salud y al ambiente. Rev Biomed 1994;5:180-190.

5. Sistema N acional de Vigilancia Epidemiológica, Secretaría de Salud. Intoxicación por plaguicidas. Boletín Epidemiología. 1996;13(50):17.

6. Sistema Nacional de Vigilancia Epidemiológica, Secretaría de Salud. Intoxicación por plaguicidas. Boletín Epidemiología. 1998;14(53):18.

7. Concha A, Guerrero R, Phebol, Migliónico A.Vigilancia epidemiológica de homicidios y suicidios. Informe del Taller de Cali. Bol O ficina Sanit Panam 1996;120:316-324.

8. Borges G, Rosovsky H, Gómez C , Gutiérrez R. Epidemiología del suicidio en México de 1970 a 1994. Salud Publica Mex 1996;38:197-206.

9. Nicholls ES. Diferenciales de mortalidad en las enfermedades no transmisibles según el nivel socioeconómico: el caso de América Latina. Bol 0 ficina Sanit Panam 1993;115:255-269.

10. Andrade $C$ arvalho W. Fatores de riscos relacionados com exposiçao ocupacional e ambiental a inseticidas organoclorados no Estado da Bahia, Brasil, 1985. Bol O ficina Sanit Panam 1991;111:512:524.

11. McConnell R, Hruska AJ. An Epidemic of pesticide poisoning in $\mathrm{N}$ icaragua: Implications for prevention in developing countries.Am J Public Health 1993;83:1559-1562.

12. Câmara V de M, Corey G.Vigilância epidemiológica relacionada com substâncias de uso proibido na agricultura. Bol 0 ficina Sanit Panam 1995; 119:135-139. 